\title{
Fertility-sparing Radiation and Chemotherapy
}

\author{
Rakesh Kapoor \\ Keywords: Brachytherapy, Chemotherapy, Fertility preservation, Radiation therapy. \\ Journal of Postgraduate Medicine Education and Research (2019): 10.5005/jp-journals-10028-1340
}

\section{INTRODUCTION}

Owing to the increasing success of oncologists over the past four decades, patients diagnosed with cancer are surviving longer, and care now focuses on improving quality of life and long-term health. Chemotherapy and radiation, the standards of care in cancer treatment, result in significant gonadotoxicity thereby impairing a woman's (and man's) fertility. As a result, cancer patients in their reproductive years are faced with another life crisis in the form of preserving their fertility.

Over 1,00,000 women 45 years of age and younger are annually diagnosed with cancer. Between 1990 and 2008, overall cancer death rates decreased by $23 \%$ in men and $15 \%$ in women, representing approximately one million lives saved. Approximately $77 \%$ of cancer patients diagnosed younger than 45 years. These rates are continuing to improve for the four most serious cancers: lung, colon, breast, and prostate. Gynecologic malignancies account for 1.09 million new cancer cases worldwide consisting of about $12 \%$ of tumors affecting female population. About $10 \%$ of all female cancer survivors are younger than 40 years of age. Since cancers affecting female genital organs are usually treated by radical surgery, chemotherapy or chemoradiation approaches that induce permanent damage of reproductive functions, the development of strategies for fertility preservation represent one of the most important goals for gynecologic oncology. In this scenario, the newly defined oncofertility discipline acquires increasing interest, offering patients maximal chances to make an adequate decision about future fertility, based on their oncologic diagnosis and prognosis. However, the majority of physicians do not pay particular attention to these issues, even if impressive progresses have been made in this field in the last decades. Possibly, it is due to the lack of strong evidences from clinical trials without an adequate number of cases to establish safety and efficacy of these procedures. In this review I will discuss the most recently debated options for fertility preservation in gynecologic oncology, with radiotherapy techniques and chemotherapy.

Cervical cancer is one of the most common cancers diagnosed in female patients under the age of 40 years. ' Successful treatment leading to cure is the major concern for most patients. However, for young patients, preservation of fertility and pregnancy related complications after treatment are also of importance. Therefore, if present, the desire to cure the cancer and additionally achieve fertility preservation poses several important considerations both for the patient and the interdisciplinary oncologic team. Due to the trend of delaying child bearing in the interest in fertility preservation might be rising in female cancer patients. For patients with cervical cancer who have to undergo chemo radiation, preservation of ovarian function and preservation of
Department of Radiotherapy, Regional Cancer Centre, Postgraduate Institute of Medical Education and Research, Chandigarh, India

Corresponding Author: Rakesh Kapoor, Department of Radiotherapy, Regional Cancer Centre, Postgraduate Institute of Medical Education and Research, Chandigarh, India, Phone: +91 9914209396, e-mail: drkapoor.r@gmail.com

How to cite this article: Kapoor R. Fertility-sparing Radiation and Chemotherapy. J Postgrad Med Edu Res 2019;53(4):168-172.

Source of support: Nil

Conflict of interest: None

the functionality of endometrial and myometrial structures are of importance but remain a challenge in clinical practice. However bilateral-oophorectomy is not part of uterus to receive nidation and to accommodate normal growth of the foetus to term. ${ }^{2}$ The non renewable pool of ovarian primordial follicles declines through atresia with age, from around 2 million at birth to 5,00,000 at menarche. Further decrease of the number of primordial follicles is associated with an increased difficulty of spontaneous conception during lifetime. ${ }^{3,4}$ This natural decrease can be aggravated by chemotherapy as well as radiation therapy causing direct DNA damage to follicles. Ovarian tissue is very sensitive to radiation. ${ }^{5}$ It was estimated that $\leq 2$ Gy will destroy half of immature oocytes ${ }^{4,6}$ and 4 Gy produces infertility in a third of young women and in almost all women over 40 years of age. ${ }^{7}$ Childhood Cancer Survivor Study (CCSS) demonstrated that the occurrence of acute ovarian failure was not only associated with older age at diagnosis but also with the conduction of abdominal or pelvic radiation therapy, especially those who received at least $10 \mathrm{~Gy}$ to the ovaries. ${ }^{8}$ Overcoming these problems would offer selected patients the chance for both, cancer control and preservation of fertility, including nidation of the ovule in their own uterus, e.g., carrying a child to term. Recent interdisciplinary approaches need to be incorporated in the management of young cancer patients desirous of preserving their fertility without comprising on the primary treatment outcome.

\section{Preservation of Ovarian Function, Cryoconservation and Ovarian Transposition}

A successful pregnancy is dependent upon a functional hypothalamic-pituitary-ovarian axis and the ability of the preservation of ovarian function is an emerging medical, emotional and quality of life issue for pre-menopausal women affected by 
cervical cancer. ${ }^{9}$ However, methods of ovarian preservation are often underused (only in 31 out of 108 patients) as demonstrated by Han et al. in a retrospective, single center study. ${ }^{10}$ Ovarian function can be preserved either by cryoconservation and re-transplantation of ovarian tissue after oncologic treatment or by ovarian transposition (OT). In current practice a proportion of young cervical cancer patients undergo cryoconservation of unfertilized oocytes after appropriate ovarian stimulation. ${ }^{11}$ Another established option which however requires a partner is in vitro fertilization (IVF) and cryopreservation of embryos, which is not regulated by legislation in several countries. ${ }^{9}$ Alternatively ovarian tissue might be cryopreserved and later be re-implanted, preferably by an orthotropic approach, a procedure which requires no partner and no hormonal stimulation. ${ }^{12}$ Whether ovarian suppression through treatment with gonodotropin-releasing hormone $(\mathrm{GnRH})$ agonists or antagonists during chemotherapy might help to maintain fertility is controversially discussed. ${ }^{13}$ First live birth after cryopreservation of ovarian tissue followed by transplantation was described in 2004 in a woman with Hodgkin's lymphoma. ${ }^{12}$ Literature review suggests that until today the birth of 18 healthy babies has been reported after transplantation of frozen-thawed human ovarian tissue. ${ }^{14}$ This promising fertility preservation strategy has also been described in a couple of young women affected by early cervical cancer. ${ }^{15,16}$ In order to reduce the dose applied to the ovaries, ovarian transplantation is a surgical procedure to move the ovaries and fallopian tube outside the radiation volume by suturing them within the paracolic gutter as high and lateral as possible (Hwang et al.) ${ }^{17}$ demonstrated that fixation more than $1.5 \mathrm{~cm}$ above iliac crest was the most important factor for intact ovarian function. ${ }^{18}$ Ovarian transplantation can be done during open radical hysterectomy, by laparoscopic approach or more recently used robot-assisted technique. ${ }^{19,20}$ Therefore, maintaining of hormonal function can be achieved in $70-93 \%$ of women younger than 40 years. ${ }^{21-26}$ Successful deliveries after IVF stimulated oocytes retrieval from transposed ovary and transfer to surrogate mothers have been described in patient treated for cervical cancer. ${ }^{27-29}$ However, metastases in transposed ovaries also may occur occasionally. ${ }^{30-32}$ Data for prevalence of ovarian metastases in patients with cervical cancer in the literature vary between $0 \%$ and $15 \%$. Known risk factors for ovarian spread are tumor size, histological type (squamous vs adenocarcinoma), grading, lymphovascular space involvement and hemovascular involvement, all of those having been discussed controversially. ${ }^{24,33-36}$

In case of other pelvic malignancies viz. sarcomas, carcinoma rectum, bone tumors, etc. occurring in young females who are desirous of fertility preservation, it is best to try to keep one or both the ovaries out of the radiation fields if possible depending upon the individual case to case with the help of sophisticated external beam irradiation techniques (intensity modulated radiation therapy [IMRT], volumetric arc therapy and helical tom therapy) offering by means of "dose painting" and sharp dose gradients against normal tissue a considerable dose reduction not only to the transposed ovaries but also to the uterus itself (Figs 1 to 6).

When IMRT should be used to spare healthy uterine tissue, an appropriate management of uterine motion is crucial, as interfractional uterine movement has been well described by others. Besides bladder and rectum filling recommendations we recommend daily soft-tissue imaging with correction for interfractional motion or adaptive replanting if deemed necessary. With the use of MRI guided brachytherapy, the extent of the macroscopic tumor can be exactly determined and the uninvolved corpus uteri should not be part of the target volume.

\section{Chemotherapy-related Ovarian Failure}

Another reason of ovarian failure might be the application of chemotherapy in combination with radiation therapy. Most of the available literature on use of chemotherapy and consecutive infertility is limited because of reporting amenorrhea as a surrogate measure of infertility. Generally, a decrease of the total number of primordial follicles could be detected after application of chemotherapeutic drugs and it appears that alkylating agents have the highest risk of permanent amenorrhea, while the risk after cisplatin-containing chemotherapy which is the drug of choice in the treatment of cervical cancer, is considered to be of intermediate risk for infertility. ${ }^{2,13}$ Furthermore, it has been described that multiagent chemotherapy without radiation therapy was not associated with the occurrence and outcome of pregnancies. ${ }^{37}$

\section{Modern Ovarian and Uterine-sparing Techniques in Radiation Oncology}

Current prechemoradiation fertility preserving strategies such as cryoconservation of oocytes or ovarian tissue and limitation of the dose applied to the ovaries, ${ }^{3}$ ultimately were depending on the use a surrogate mother, as uterine dysfunction after pelvic radiation therapy was assumed to preclude to carry a pregnancy to term. However, due to the availability of newer radiation therapy techniques including IMRT as well as CT and MRT based application of cervical HDR-brachytherapy or even HDR-brachytherapy emulating strategies, e.g., using robotic radio surgery, along with improved fertility preservation methods by reproductive medicine experts, today, the question arises whether fertility can be preserved in young patients with cervical cancer including the ability to carry a pregnancy to term. This would have also forensic implications as third-party reproduction using a gestational carrier is illegal in several European countries. The radio sensitivity of the uterus appears to decrease with advanced age as mentioned above but less data is available from the literature regarding acute and late radiation dose effects on the adult uterus. Milgrom et al. ${ }^{38}$ recently described the acute uterine effects after pelvic radiation therapy with a median dose of $50.2 \mathrm{~Gy}$ (D95 of the uterus was $30 \mathrm{~Gy}$ ) in 10 female (7 of which were pre-menopausal) rectal cancer patients who underwent dynamic contrast-enhanced MRI before and 4-7 weeks after radiation therapy. It was found that the median cervical length was reduced after radiation therapy. Interestingly three of the analyzed patients who were initially premenopausal underwent ovarian transposition and maintained ovarian function after radiation therapy and three other patients were postmenopausal before radiation therapy. Thus in these six patients radiation induced ovarian failure would not account for the changes in uterine anatomy. Moreover, in pre-menopausal patients the volume transfer constant (Ktrans) and the extracellular extra vascular volume fraction (Ve) were significantly decreased after radiation therapy, suggesting reduced perfusion of the premenopausal myometrium after radiation therapy. ${ }^{38}$

These functional changes of the uterus could both lead to an impaired implantation of an embryo as well as pregnancy-related complications. ${ }^{3}$ The degree of damage has been shown to be dependent on the total radiation dose and it was shown that the pre-pubertal uterus is more vulnerable than 
the adult uterus to the effect of pelvic radiation therapy, with doses of 14-30 Gy causing uterine dysfunction. ${ }^{3,39,40}$ It has been reported after total body irradiation using 8.5-11.7 Gy total dose ${ }^{41}$ or 14.4 Gy total dose (2.40) in young female patients, that uterine growth

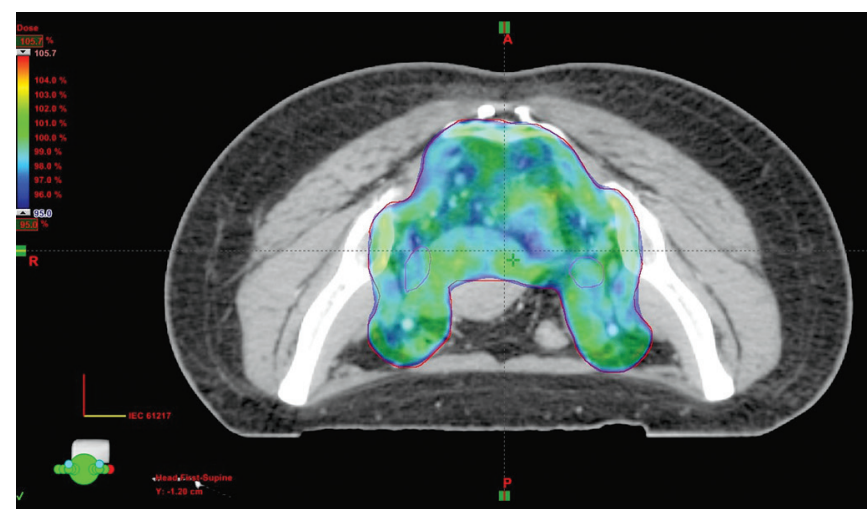

Fig. 1: VMAT plan with color wash

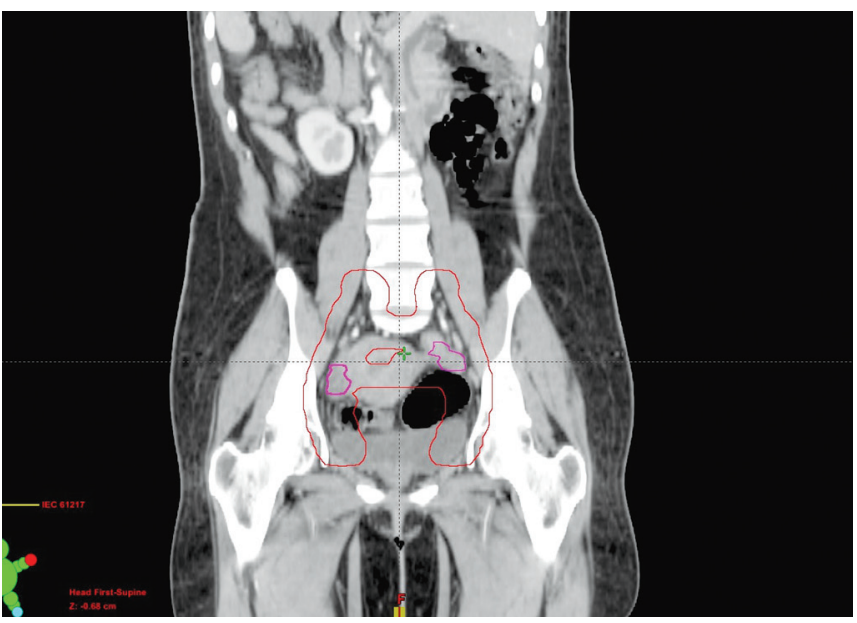

Fig. 3: VMAT plan showing ovarian sparing and blood flow were impaired. Likewise, after whole-abdominal radiation therapy using 20-30 Gy during childhood the uterine length was shorter and endometrial thickness was not increased after hormone replacement suggesting irreversible damage to the uterus. ${ }^{39}$ Others have described in a cohort of 340 female cancer survivors that after abdominopelvic radiation therapy the likelihood to have low-birth-weight infants, premature low-birth-weight infants and the parental infant mortality was increased as compared to patients without radiation therapy. These associations were dose

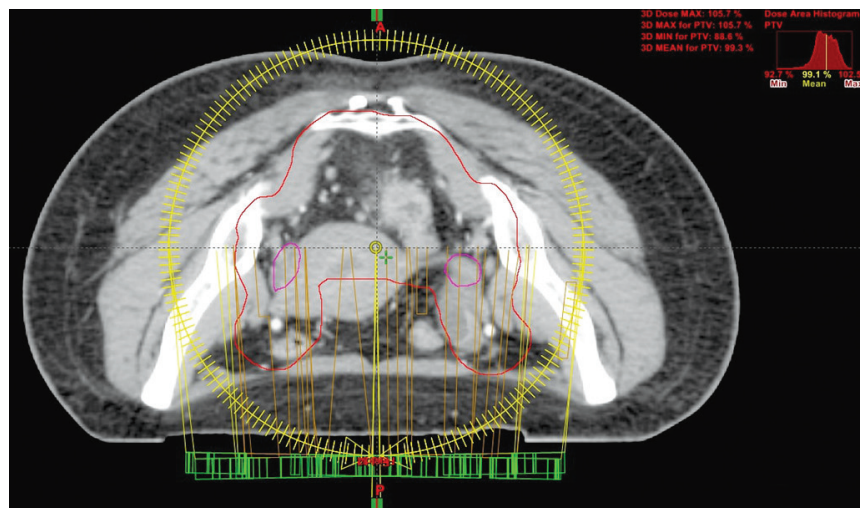

Fig. 2: VMAT plan showing ovarian sparing

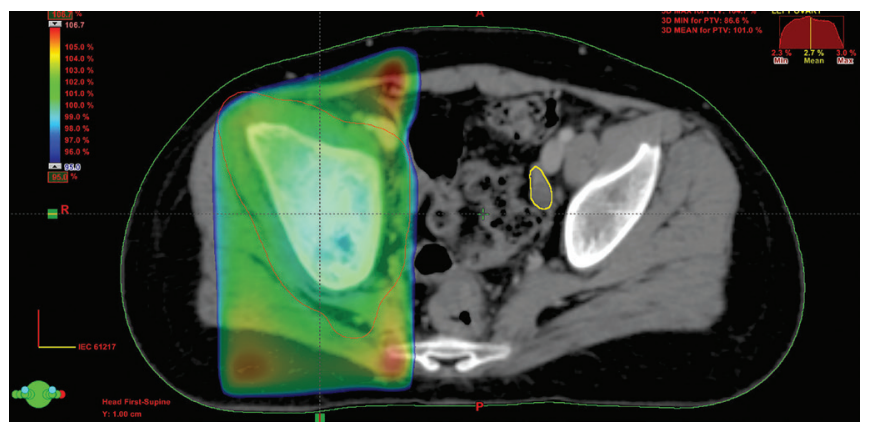

Fig. 4: Dose color wash showing sparing of left ovary

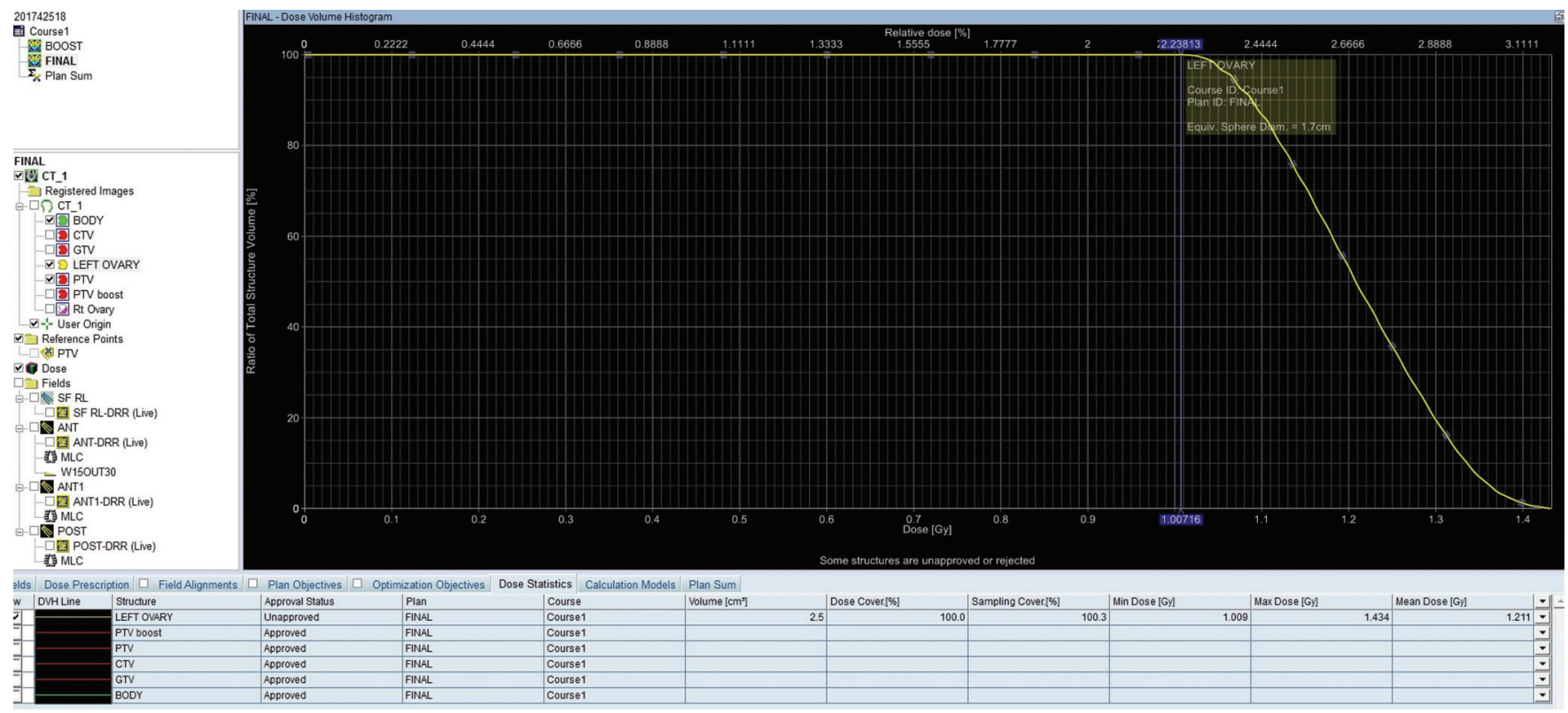

Fig. 5: DVH chart showing approved plan 


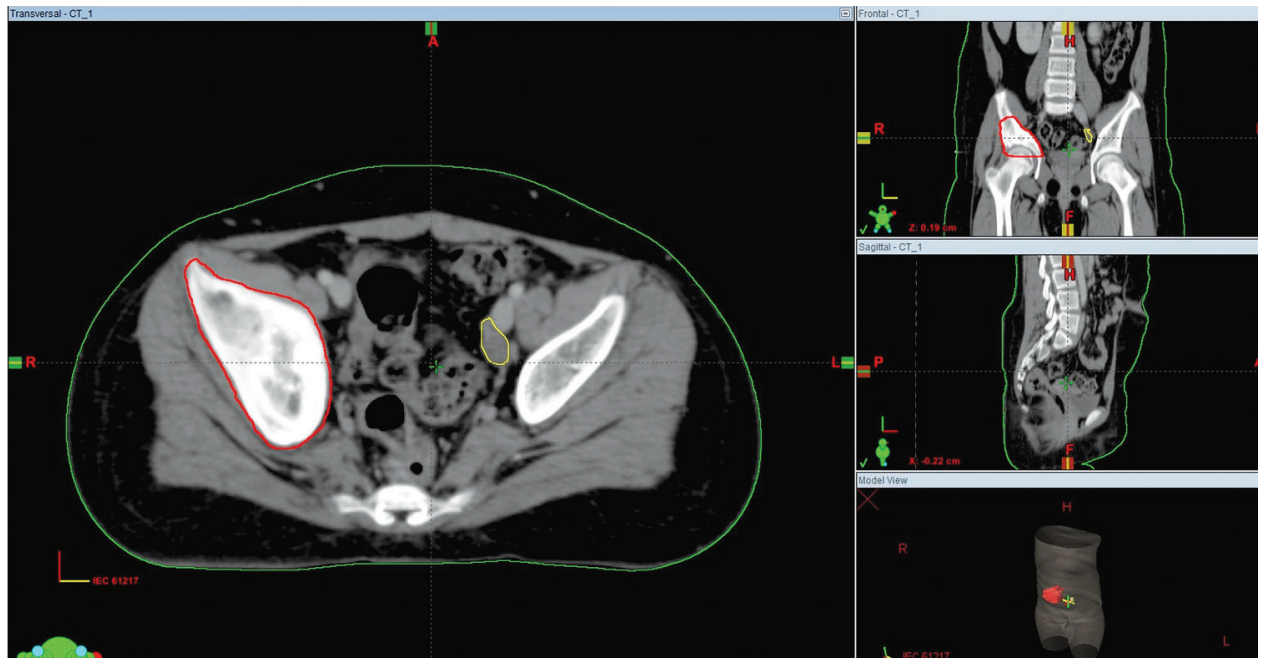

Fig. 6: Showing contouring of GTV of right iliac bone tumor (red ) and left ovary (yellow)

dependent and the likelihood to have low-birth-weight infants and parental infant mortality were higher in patients receiving $>25$ Gy as compared to total doses below $25 \mathrm{~Gy}$. ${ }^{42}$

\section{Conclusion}

Fertility preservation options depend on many factors. The age of the patient will provide insight to her ovarian reserve to contemplate the utility of fertility preservation. The tumor type, stage, and treatment plan determine the time available, if any, to proceed with an emergency IVF cycles.

Younger than age 40 undergoing chemotherapy will experience ovarian failure following treatment. Alkylating agents, particularly the combination of oral cyclophosphamide, methotrexate, and fluorouracil (CMF) have the highest risk of ovarian failure. Post chemotherapy resumption of menses, if applicable, occurs in 6 months but may require up to 2 years. It is important to note that the return of menstrual function does not equate with maintenance of pretreatment biologic ovarian age. Therapy results in the death of primordial ovarian follicles and interrupts follicle recruitment and maturation, resulting in decreasing ovarian reserve. So, the reproductive potential of a woman post chemotherapy can still be impaired despite the return of menstrual cycles as demonstrated by a higher rate of infertility and lower ovarian reserve. Furthermore, even prior to therapy, cancer patients have a lower number of eggs retrieved after gonodotropin stimulation for fertility preservation than age-matched healthy controls. ${ }^{3}$ Patients undergoing pelvic and abdominal irradiation are at significant risk for ovarian failure following treatment. The ovarian follicles are remarkably sensitive to DNA damage from ionizing radiation. The most damage is from single-dose total radiation therapy rather from fractionated therapy. A dose above $300 \mathrm{cGy}$ is the threshold for permanent ovarian failure. Most pelvic malignancies and Hodgkin lymphoma require radiation doses over, 1,000 cGy and are associated with the highest risk for permanent loss of ovarian function.

\section{References}

1. Siegel R, Naishadham D, Jemal A. Cancer statistics, 2013. CA Cancer J Clin 2013;63(1):11-30. DOI: 10.3322/caac.21166.

2. Critchley HO, Wallace WH. Impact of cancer treatment on uterine function. J Natl Cancer Inst Monogr 2005;2005(34):64-68. DOI: 10.1093/jncimonographs/lgi022.
3. Wo JY, Viswanathan AN. Impact of radiotherapy on fertility, pregnancy, and neonatal outcomes in female cancer patients. Int J Radiat Oncol Biol Phys 2009;73(5):1304-1312. DOI: 10.1016/j. ijrobp.2008.12.016.

4. Faddy MJ, Gosden RG, Gougeon A, et al. Accelerated disappearance of ovarian follicles in mid-life: implications for forecasting menopause. Hum Reprod 1992;7(10):1342-1346. DOI: 10.1093/oxfordjournals. humrep.a137570.

5. Gross E, Champetier C, Pointreau Y, et al. Normal tissue tolerance to external beam radiation therapy: ovaries. Cancer Radiother 2010;14(4-5):373-375. DOI: 10.1016/j.canrad.2010.01.011.

6. Wallace $\mathrm{WH}$, Thomson AB, Kelsey TW. The radio sensitivity of the human oocyte. Hum Reprod 2003;18(1):117-121. DOI: 10.1093/ humrep/deg016.

7. Oncology RotJCfC. Management of Gonadal Toxicity Resulting from the Treatment of Adult Cancer; 1998. ISBN 1860160719.

8. Chemaitilly W, Mertens AC, Mitby P, et al. Acute ovarian failure in the childhood cancer survivor study. J Clin Endocrinol Metab 2006;91(5):1723-1728. DOI: 10.1210/jc.2006-0020.

9. Oktay K. Further evidence on the safety and success of ovarian stimulation with letrozole and tamoxifen in breast cancer patients undergoing in vitro fertilization to cryopreserve their embryos for fertility preservation. J Clin Oncol 2005;23(16):3858-3859. DOI: 10.1200/JCO.2005.04.011.

10. Han SS, Kim YH, Lee SH, et al. Underuse of ovarian transposition in reproductive-aged cancer patients treated by primary or adjuvant pelvic irradiation. J Obstet Gynaecol Res 2011;37(7):825-829. DOI: 10.1111/j.1447-0756.2010.01443.x.

11. Anderson RAWW. Fertility preservation in girls and young females. Clin Endocrinol (Oxf) 2011;75(4):409-419. DOI: 10.1111/j.13652265.2011.04100.x.

12. Donnez J, Dolmans MM, Demylle D, et al. Livebirth after orthotopic transplantation of cryopreserved ovarian tissue. Lancet 2004;364(9443):1405-1410. DOI: 10.1016/S0140-6736(04)17222-X.

13. Lee SJ, Schover LR, Partridge AH, et al. American Society of Clinical Oncology recommendations on fertility preservation in cancer patients. J Clin Oncol 2006;24(18):2917-2931. DOI: 10.1200/ JCO.2006.06.5888.

14. Dittrich R, Lotz $L$, Keck $G$, et al. Live birth after ovarian tissue autotransplantation following overnight transportation before cryopreservation. Fertil Steril 2012;97(2):387-390. DOI: 10.1016/ j.fertnstert.2011.11.047.

15. Kim SS, Hwang IT, Lee HC. Heterotopic autotransplantation of cryo banked human ovarian tissue as a strategy to restore ovarian function. Fertil Steril 2004;82(4):930-932. DOI: 10.1016/j.fertnstert.2004. 02.137 . 
16. Kim SS. Assessment of long term endocrine function after transplantation of frozen-thawed human ovarian tissue to the heterotopic site: 10 year longitudinal follow-up study. J Assist Reprod Genet 2012;29(6):489-493. DOI: 10.1007/s10815-012-9757-3.

17. Clough KB, Goffinet F, Labib A, et al. Laparoscopic unilateral ovarian transposition prior to irradiation: prospective study of 20 cases. Cancer 1996;77(12):2638-2645. DOI: 10.1002/(SICI)10970142(19960615)77:12<2638::AID-CNCR30>3.0.CO;2-R.

18. Hwang $\mathrm{JH}$, Yoo HJ, Park $\mathrm{SH}$, et al. Association between the location of transposed ovary and ovarian function in patients with uterine cervical cancer treated with (postoperative or primary) pelvic radiotherapy. Fertil Steril 2012;97(6):1387-1393. e1381-e1382 10.1016/ j.fertnstert.2012.02.052.

19. Morice $P$, Juncker L, Rey $A$, et al. Ovarian transposition for patients with cervical carcinoma treated by radiosurgical combination. Fertil Steril 2000;74(4):743-748. DOI: 10.1016/S0015-0282(00)01500-4.

20. lavazzo C, Darlas FM, Gkegkes ID. The role of robotics in ovarian transposition. Acta Inform Med 2013;21(2):135-137. DOI: 10.5455/ aim.2013.21.135-137.

21. Gallocher O, Thomas L, Stockle E, et al. First surgery followed by vaginal curie therapy in small-volume uterine cervix cancer: an alternative to the association of utero vaginal curie therapy and surgery. Cancer Radiother 2002;6(1):10-14.

22. Barahmeh S, Al Masri M, Badran O, et al. Ovarian transposition before pelvic irradiation: indications and functional outcome. J Obstet Gynaecol Res 2013;39(11):1533-1537. DOI: 10.1111/jog.12096.

23. Olejek $A$, Wala $D$, Chimiczewski $P$, et al. Hormonal activity of transposed ovaries in young women treated for cervical cancer. Gynecol Endocrinol 2001;15(1):5-13. DOI: 10.1080/gye.15.1.5.13.

24. Huang KG, Lee $C L$, Tsai CS, et al. A new approach for laparoscopic ovarian transposition before pelvic irradiation. Gynecol Oncol 2007;105(1):234-237. DOI: 10.1016/j.ygyno.2006.12.001.

25. Ishii K, Aoki Y, Takakuwa K, et al. Ovarian function after radical hysterectomy with ovarian preservation for cervical cancer. J Reprod Med 2001;46(4):347-352.

26. Pahisa J, Martinez-Roman S, Martinez-Zamora MA, et al. Laparoscopic ovarian transposition in patients with early cervical cancer. Int J Gynecol Cancer 2008;18(3):584-589. DOI: 10.1111/j.15251438.2007.01054.x.

27. Giacalone PL, Laffargue F, Benos $\mathrm{P}$, et al. Successful in vitro fertilizationsurrogate pregnancy in a patient with ovarian transposition who had undergone chemotherapy and pelvic irradiation. Fertil Steril 2001;76(2):388-389. DOI: 10.1016/S0015-0282(01)01895-7.

28. Zinger M, Liu JH, Husseinzadeh N, et al. Successful surrogate pregnancy after ovarian transposition, pelvic irradiation and hysterectomy. J Reprod Med 2004;49(7):573-574.

29. Agorastos T, Zafrakas M, Mastrominas M. Long-term follow-up after cervical cancer treatment and subsequent successful surrogate pregnancy. Reprod Biomed Online 2009;19(2):250-251. DOI: 10.1016/ S1472-6483(10)60080-6.
30. Delotte J, Ferron G, Kuei TL, et al. Laparoscopic management of an isolated ovarian metastasis on a transposed ovary in a patient treated for stage IB1 adenocarcinoma of the cervix. J Minim Invasive Gynecol 2009;16(1):106-108. DOI: 10.1016/j.jmig.2008.10.003.

31. Shigematsu T, Ohishi $Y$, Fujita $T$, et al. Metastatic carcinoma in a transposed ovary after radical hysterectomy for a stage $1 \mathrm{~B}$ cervical adenosquamous cell carcinoma. Case report. Eur J Gynaecol Oncol 2000;21(4):383-386.

32. Morice P, Haie-Meder C, Pautier P. Ovarian metastasis on transposed ovary in patients treated for squamous cell carcinoma of the uterine cervix: report of two cases and surgical implications. Gynecol Oncol 2001;83(3):605-607. DOI: 10.1006/gyno.2001.6447.

33. Zhao C, Wang JL, Wang SJ, et al. Analysis of the risk factors for the recurrence of cervical cancer following ovarian transposition. Eur J Gynaecol Oncol 2013;34(2):124-127.

34. Nakanishi T, Wakai K, Ishikawa $\mathrm{H}$, et al. A comparison of ovarian metastasis between squamous cell carcinoma and adenocarcinoma of the uterine cervix. Gynecol Oncol 2001;82(3):504-509. DOI: 10.1006/ gyno.2001.6316.

35. Shimada M, Kigawa J, Nishimura R, et al. Ovarian metastasis in carcinoma of the uterine cervix. Gynecol Oncol 2006;101(2):234-237. DOI: 10.1016/j.ygyno.2005.10.004.

36. Yamamoto R, Okamoto K, Yukiharu T, et al. A study of risk factors for ovarian metastases in stage Ib-IIlb cervical carcinoma and analysis of ovarian function after a transposition. Gynecol Oncol 2001;82(2):312-316. DOI: 10.1006/gyno.2001.6277.

37. Green DM, Whitton JA, Stovall M, et al. Pregnancy outcome of female survivors of childhood cancer: a report from the Childhood Cancer Survivor Study. Am J Obstet Gynecol 2002;187(4):1070-1080. DOI: 10.1067/mob.2002.126643.

38. Milgrom SA, Vargas HA, Sala E, et al. Acute effects of pelvic irradiation on the adult uterus revealed by dynamic contrastenhanced MRI. Br J Radiol 2013;86(1031):20130334. DOI: 10.1259/bjr. 20130334.

39. Critchley HO, Wallace $W H$, Shalet $\mathrm{SM}$, et al. Abdominal irradiation in childhood; the potential for pregnancy. $\mathrm{Br} J$ Obstet Gynaecol 1992;99:392-394. DOI: 10.1111/j.1471-0528.1992.tb13755.x.

40. Bath LE, Critchley HO, Chambers SE, et al. Ovarian and uterine characteristics after total body irradiation in childhood and adolescence: response to sex steroid replacement. $\mathrm{Br} J$ Obstet Gynaecol 1999;106(12):1265-1272. DOI: 10.1111/j.1471-0528.1999. tb08180.x.

41. Holm K, Nysom K, Brocks V, et al. Ultrasound B-mode changes in the uterus and ovaries and Doppler changes in the uterus after total body irradiation and allogeneic bone marrow transplantation in childhood. Bone Marrow Transplant 1999;23(3):259-263.DOI: 10.1038/ sj.bmt.1701569.

42. Chiarelli AM, Marrett LD, Darlington GA. Pregnancy outcomes in females after treatment for childhood cancer. Epidemiology 2000;11(2):161-166. DOI: 10.1097/00001648-200003000-00013. 